\title{
A Novel Energy-Efficient Heuristic for Target Coverage to Maximize Sensor Network Lifetime
}

\author{
Manju \\ ITM University \\ Huda Sector 23A \\ Gurgaon
}

\author{
Anuradha \\ ITM University \\ Huda Sector 23A \\ Gurgaon
}

\begin{abstract}
To achieve power-efficient monitoring of targets in a terrain covered by a sensor network, it is sensible to divide the sensors into cover sets and make each of these sets responsible for covering the targets for a certain period of time. Generating the maximum number of such cover sets has been proved to be an NP-complete problem, and thus algorithms producing suboptimal solutions have been proposed. This paper proposes an efficient method to extend the sensor network operational time by organizing the sensors into a maximal number of non-disjoint sensor covers that are activated successively. Only the sensors from the current active sensor cover are responsible for monitoring all targets and for transmitting the collected data, while nodes from all other sensor covers are in a low-energy sleep mode. It first discus the problems associated with existing heuristic for the target coverage and then this paper proposes a new solution to maximize total network lifetime.
\end{abstract}

\section{General Terms}

Algorithm, target coverage problem, wireless sensor networks

\section{Keywords}

Heuristic, NP completeness, Wireless Sensor Networks, Network lifetime

\section{INTRODUCTION}

Wireless Sensor Networks (WSNs) are used extensively in various domains, such as military applications [1], environmental monitoring [14], target surveillance [13] and disaster prevention [11]. A wireless sensor network consists of a large number of tiny sensor nodes to accomplish a large sensing task. Sensor nodes are small devices equipped with one or more sensors, one or more transceivers, processing, storage resources and possible actuators [11]. Sensors in a network can cooperatively gather information from an interest region of observation and transmit this collected information to a base station. As each sensor node is equipped with at least one sensing module and one communication module, which enable the collection of monitoring data and their transmission to a base station, which is situated in close proximity to the WSN. The base station may be a desktop computer or a laptop. Sensor nodes organize in networks and collaborate to accomplish a larger sensing task. The characteristics of a sensor network include limited resources, large and dense networks (of hundreds or even thousands of sensor nodes) and a dynamic topology.

During the usages of a sensor node, at a time it can be in four different states: transmit, receive, idle, or sleep [16]. The idle state is when the transceiver is neither transmitting nor receiving, and the sleep mode is when the radio is turned off. During the transmit state, a sensor node is responsible to transmit the collected data/information to the designated place. In the receive state, a sensor node is about to receive complete data which is incident in the given sensor range. Judiciously selecting the state of each sensor node's radio is accomplished through a scheduling mechanism.

Energy-efficiency is an important issue in WSN, because battery resources are limited. Mechanisms that conserve energy resources are highly desirable, as they have a direct impact on network lifetime. Network lifetime is in general defined as the time interval the network is able to perform the sensing functions and to transmit data to the sink. During the network lifetime, some nodes may become unavailable (e.g. physical damage, lack of power resources) or additional nodes might be deployed. One of the most prominent methods is based on the scheduling sensor activity so that a set of active sensors can handle a required task while the rest of the redundant sensors can enter into sleep mode.

The battery consumption of the sensors needs to be managed efficiently to extend the network lifetime. Energy-efficient monitoring of targets can be achieved by dividing the sensors into subsets called sensor cover responsible for covering all the targets for certain period of time. Sensor cover is the subsets of sensors that cover all the targets. Here, objective is to schedule the sensor covers in such a way that only one cover is active at a time to cover all the targets. So, instead of activating all the sensor covers, a subset of sensors is active, thus reducing power consumption and extending network lifetime.. If the sensor covers are non-disjoint sets, then each sensor will be allowed to participate in more than one cover set. By periodically switching between sensor covers of coverage sensors, and this extend the total network lifetime.

This paper addresses the sensor scheduling mechanism that does not allow all the sensor nodes to enter in the active mode. To design such a mechanism, one must handle some situations those can be raised as follows:

1) Which criteria should be followed by each node to determine their mode of operation (active/sleep mode)?

\section{2) At what time a node should make such a decision?}

\section{3) How long should a sensor remain in the sleep mode?}

The sensor coverage problem is an important problem in wireless sensor networks (WSN). Here the main goal is to have each location (targets) in the interested physical space within the sensing range of at least one sensor. In wireless sensor networks, sensors are densely deployed to cover the area completely. Thus it is possible to turn some sensors while guaranteeing the complete coverage of the interest region. One of the main objectives of the coverage problem is to prolong network lifetime. Besides this objective, the coverage problem has many variants based on the other constraints of networks and objects to be covered. Based on a survey on sensor coverage problems in wireless sensor networks [6], the 
coverage problems are classified in the following types [4]: (1) area coverage [10], [13], [18], [19], [20], where the objective is to cover an area, (2) point coverage [5], [4], [7], where the objective is to cover a set of targets, and (3) coverage problems that have the objective to determine the maximal support/breach path that traverses a sensor field [15].

There are many algorithms proposed [2, 4, 12, 21] to devise such a mechanism to organize the sensors into a number of subsets such that each subset can completely covers set of targets in order to extend network lifetime.

This paper, propose a novel centralized algorithm for the generation of non-disjoint sensor cover for target coverage problem in wireless sensor networks. Each sensor cover is capable of monitoring independently all registered targets. The algorithm provides efficient solutions for both point and area coverage problems. Furthermore, the simulation on proposed algorithm is carried out which compare its results with [5], with regard to the total time of execution and number of generated sensor covers.

\section{RELATED WORK}

Cardei and $\mathrm{Wu}[5]$ provide a detailed view of sensor coverage algorithms according to several design criteria, such as:

1. The coverage objective, i.e. to maximize the lifetime of the network or to minimize the required number of deployed sensors.

2. The node deployment method, which may be random or deterministic.

3. The homogeneous or heterogeneous nature of the nodes, i.e. whether all nodes have a common sensing or communication range.

4. The degree of centralization, i.e. centralized vs. distributed algorithms, and

5. Additional requirements for energy efficiency and connectivity.

An additional criterion that can be added to the above list is the cover set independence, i.e. whether a node appears in exactly one of the generated sets (as in the case of node disjoint coverage algorithms) or not (as in the case of nondisjoint coverage algorithms). The following section present an overview of above said categories for providing coverage under various design issues.

\section{Centralized and Distributed Coverage Algorithms}

The entire centralized coverage algorithms [3, 5, 21] first calculated the monitoring schedule on the base station and it is then sent to the sensor nodes for execution. Such scheduling approach is quite beneficial as it requires very low processing power from the sensor nodes, which usually have limited processing capabilities. Centralized coverage algorithms can be further grouped into two categories: node-disjoint and nondisjoint. This paper addresses the non-disjoint part. In case of distributed algorithms $[3,9,12]$, the decision process is decentralized and all sensor nodes perform the required task cooperatively and then they share the scheduling information to the rest of the sensors.

\section{Disjoint and Non-Disjoint Algorithms}

As the paper already discussed that for extending the sensor network lifetime through energy resource preservation is the division of the sensors into sets where each sensor can participate only in one set cover. Such sets in which each sensor can be part of only one set are known as disjoint set.
These disjoint sets are activated successively, such that at any moment in time only one set is active. The goal of the approaches $[8,17,21]$ is to determine a maximum number of disjoint sets, so that the time interval between two activations for any given sensor is longer.

To increase the total network lifetime of WSN the nondisjoint algorithms are proven to be better than disjoint algorithms. While working under non-disjoint approach, nodes can participate in more than one sensor cover. In some cases, this may prolong the lifetime of the network in comparison to the disjoint cover set algorithms, but it incurs a higher complexity. Cardei et al. [5] propose a Linear Programming (LP) solution to the target coverage problem for non-disjoint cover sets. Although the LP algorithm presents a high complexity $\mathrm{O}\left(\mathrm{C}^{3} \mathrm{n}^{3}\right)$, where $\mathrm{C}$ is the number of covers and $n$ the number of sensors, the authors also propose a greedy algorithm, with a lower complexity $\mathrm{O}\left(\mathrm{dk}^{2} \mathrm{n}\right)$, where $\mathrm{d}$ is the number of sensors that cover targets that are associated with a minimum number of sensors and $\mathrm{k}$ is the number of targets. Another LP technique is proposed by Berman et al. [2]. The authors first compute a series of cover sets and then they deduce the optimal lifetime for each cover set. Their approach is based on the $(1+\varepsilon)$-approximation of the Garg and Konemann algorithm [12], with an approximation factor of (1 $+\varepsilon)(1+2 \ln n)$ for any $\varepsilon>0$.

\section{NETWORK MODEL AND PROBLEM DESCRIPTION}

Let $s_{1}, s_{2}, \ldots$, and $s_{n}$ are randomly deployed $n$ sensors and $t_{1}$, $t_{2}, \ldots$, and $t_{m}$ be $m$ targets. Each sensor $s_{i}$ is assigned a battery life of $b_{i}$. A sensor $s_{i}$ covers a target $t_{j}$ if the $t_{j}$ lies within the sensing range of $\mathrm{s}_{\mathrm{i}}$. A sensor cover $\mathrm{S}$ is a set of sensors that jointly cover all the targets. Formally, $S=\left\{s_{i} \mid\right.$ for each $t_{j}$ there is a $s_{i} \in S$ such that $s_{i}$ covers $\left.t_{j}\right\}$. The life time of a sensor cover $\mathrm{S}, \mathrm{x}(\mathrm{S})$, cannot exceed $\mathrm{Min}_{\mathrm{si} \in \mathrm{S}} \mathrm{b}_{\mathrm{i}}$.

Energy efficient target coverage problem [5] is used to maximize the total number of sensor covers. The target coverage problem presented by Cardei et al. [5] is to find a family of sensor covers (with non-zero $x(S)$ ) which has maximum aggregated lifetime among all families of covers. In other words, the network lifetime is prolonged by designing maximum number of sensor covers with the given constraints of $b_{i}$ as the battery life of sensor $s_{i}$. The problem is shown to be NP-complete [5].

\section{Definition 1 (sensor cover)}

A sensor cover $\mathrm{S}$ is a set of sensors which collectively covers all the targets.

A sensor cover $\mathrm{S}$ is a minimal cover if for any cover $\mathrm{S}^{\prime}, \mathrm{S}^{\prime} \subseteq$ $\mathrm{S}$ if and only if $\mathrm{S}^{\prime}=\mathrm{S}$.

\section{Definition 2(maximum allowable lifetime of sensor cover $S$ )}

The maximum allowable lifetime of a sensor cover $\mathrm{S}$ is the

$$
\text { max_lifetime }(S)=\operatorname{Min}{ }_{S i \in S}{ }^{b}
$$

smallest available lifetime of its sensors. Thus where $b_{i}$ is the battery life of sensor si. 


\section{Definition 3 (critical target)}

A target $t_{j}$ is said to be critical target if it is covered by minimum number of sensors. The upper bound on lifetime of sensor network is limited by the critical target only.

\section{MOTIVATION}

All the given approaches [9, 12, 17] to solve the target coverage problem are basically having same objective of maximizing total network lifetime with given limited battery life of each sensor. The heuristic solutions provided by all these authors are almost sharing same idea and simply differing in the case that how to generate sensor covers.

To propose new solution for the target coverage problem, this paper first gives an overview of generic a greedy algorithm that generates a sensor cover and assigns maximum allowable lifetime to covers. At any stage of the algorithm, bi is the residual life time of the sensor si. Below algorithm Generic Greedy Algorithm gives the pseudo code of the simple greedy heuristic.

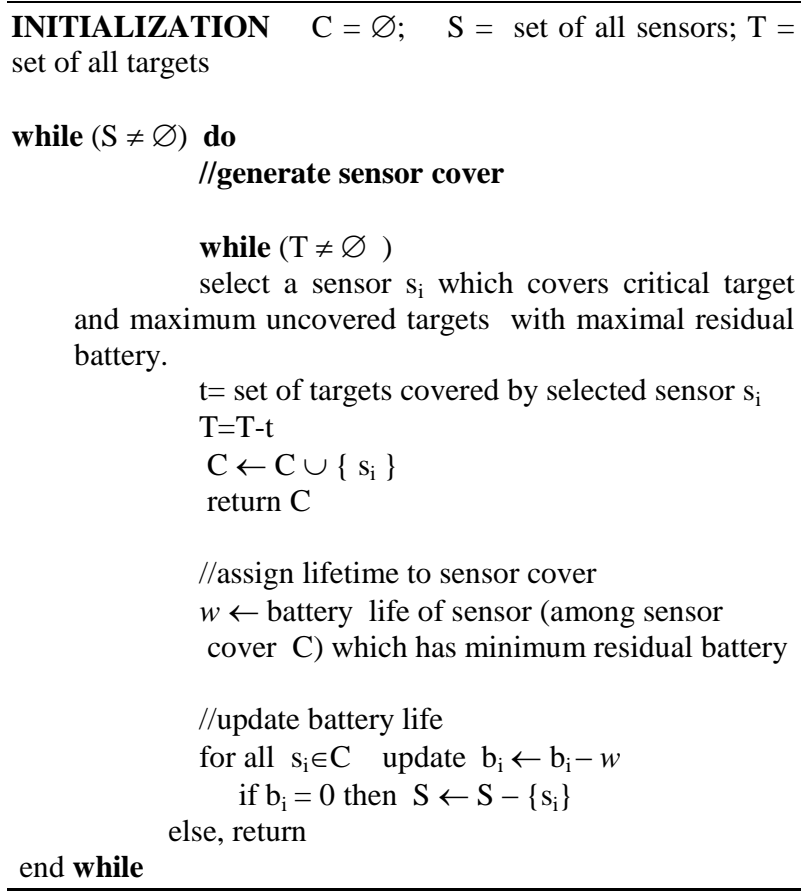

Fig 1: Generic Greedy Algorithm

As it is mentioned clearly in the Generic Greedy Algorithm that to generate a sensor cover, it always tries to give priority to that sensor which covers critical target with maximum number of uncovered target and maximal residual (energy) batter life. But, finding critical target at every step in each iteration of sensor cover generation is not possible without price. By keeping this fact in mind this paper propose a new heuristic for the target coverage problem in which the proposed heuristic avoids to find critical target and simply prioritizing sensors in terms of maximum uncovered targets. And, then always select a sensor which covers maximum uncovered targets to generate sensor cover.

\section{MAXIMUM COVERAGE FIRST (MCF)}

Based on above observations, this section comprise of a new proposed heuristic to solve the target coverage problem. The paper already discussed that sensors are having limited battery life and which is not rechargeable. So, to maximize total network lifetime, this needs to minimize total number of sensors selected in each sensor cover (maximal cover) such that all the targets are remain covered. Hence prioritizing the sensors in terms of maximal covered targets provides us better opportunity of using the sensors. MCF uses the three important steps in the following manner.

\section{Sensor Cover (Maximal Cover) generation}

The MCF heuristic generates sensor cover $C$ by selecting a sensor which covers maximum uncovered targets. Ideally, some sort of priority (coverage) is associated with each sensor. The sensor cover is constructed by iteratively selecting sensors of high priorities till all the targets are covered.

\section{Lifetime Assignment to a Maximal Cover}

For a maximal cover $\mathrm{C}$ generated in the previous step, we assign some lifetime. As shown in generic greedy, the temptation is to assign maximum allowable lifetime.

\section{Update the Battery of Sensors in Maximal Cover}

Once a sensor is selected in sensor cover, its battery life has to be reduced by the amount $w$ (assigned to the generated sensor cover).

Below is the pseudocode of proposed Maximal Coverage First (MCF).

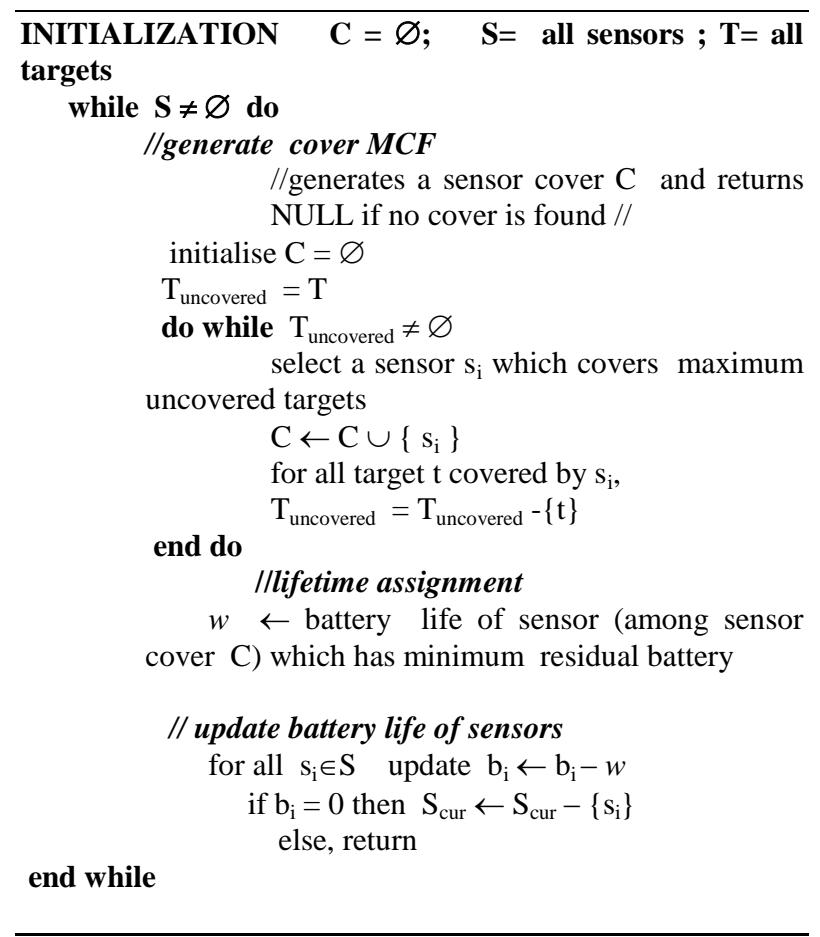

Fig 2: Maximum Coverage First

\section{SIMULATION RESULT}

For the experimental study, the proposed heuristic (MCF) is implemented the in c programming language. All experiments were carried out on a Pentium (4) processor, $2.63 \mathrm{GHz}$ host with $256 \mathrm{MB}$ of RAM, running Window Xp/Linux operating system. The heuristic generate the problem instances randomly and then assume a sensing area of $800 \times 800 \mathrm{~m}$ inside the monitored area of $1000 \times 1000 \mathrm{~m}$. For experimentation, MCF and BGOP [21] both assume a homogenous sensor networks. Here each sensor initially assigned battery (energy) equal to 1 unit and sensing range $70 \mathrm{~m}$. Sensors and targets are generated in terms of their coordinates randomly. For our experiments, MCF and BGOP [21] take sensors in the interval $[20,100]$ and the number of targets in $[20,70]$. In the following, the above said setup under simulation shows the 
performance of proposed (MCF) algorithm with existing algorithm (BGOP) proposed by Zorbas et al. [21].

\section{Experiment 1}

The experiment try to compare the performance of MCF algorithm with Zorbas et al. [21] proposed algorithm (BGOP) for the same target coverage problem. Both (MCF and BGOP) differs only in the way they selecting sensors to generate sensor cover. Zorbas et al. [21] addresses the target coverage problem with node-disjoint sensor covers. In other words, once a sensor is used in one sensor cover, it cannot be used in any other cover. When initial value of all batteries bi are equal and if this assign maximum allowable lifetime at every stage then the set of sensor covers so generated are mutually node-disjoint. Though the algorithm solves a subclass of general target coverage problem, the paper discusses this algorithm here as it follows the same paradigm. A sensor is categorized into four classes; Best, Good, OK and Poor depending on the whether it covers all (or part of) uncovered (and covered) targets. Thus, with the progress of the algorithm, the category of a sensor is getting changed. The sensor covers are greedily constructed by iteratively selecting sensors in order of Best, Good, OK and Poor.

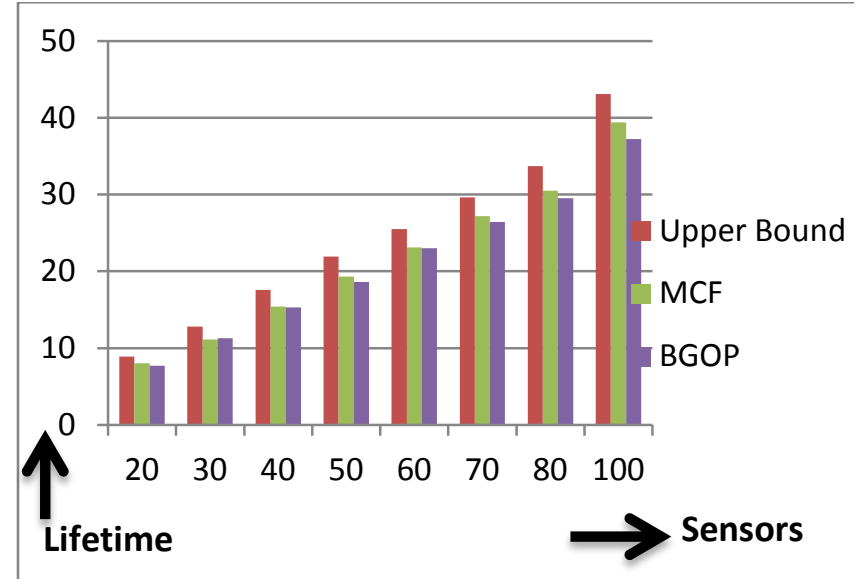

Fig 3: The average of lifetime obtained by MCF and BGOP for 15 random problem instances for different sensors and sensing range equals $70 \mathrm{~m}$.

The above Fig 3 shows the performance of MCF and BGOP on the discussed platform. For this experiment, it had taken fixed number of targets 100 and varying number of sensors in interval $[20,100]$. Here Upper Bound is maximum possible network lifetime. In Fig 3, it is clearly shown that MCF outperforms BGOP in terms of total network lifetime.

\section{Experiment 2}

The experiment in this section, compare the performance of MCF with BGOP [21] in the same setup of simulation environment. For this experiment, we considered the fixed number (100) of sensors and varying number of targets in the interval $[20,70]$. Fig 4 as given below clearly shows the total network lifetime achieved under given setup for both of the algorithms (MCF and BGOP [21]. Again it is clearly seen that MCF outperforms BGOP [21] in terms of total network lifetime gain.

Thus, above simulation shows that the newly proposed heuristic for maximizing the total network lifetime under battery constrained sensor networks always outperforms BGOP [21].

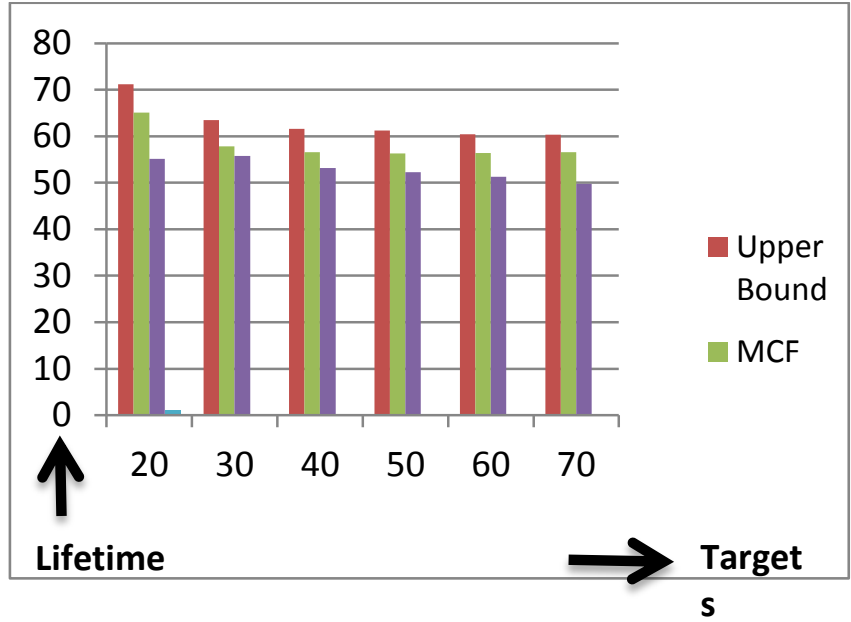

Fig 4: The average of lifetime obtained by MCF and BGOP for 15 random problem instances for fixed sensors and varying targets with sensing range equals $70 \mathrm{~m}$.

\section{CONCLUSION}

The paper had given overview of centralized algorithms for the energy-efficient target coverage problem. Though one of the algorithms makes some claim on the quality of solution, it is not a practical bound for large number of sensors. Simple, generic technique can be utilized to get a reasonably good solution. The paper proposes a new heuristic, Maximum Coverage First (MCF) which prioritizes sensors based on their coverage. The simulation results in above section shows that proposed algorithm (MCF) is performing better than one of the existing algorithm (Zorbas et al. [21]) in terms of total network lifetime.

\section{ACKNOWLEDGMENTS}

The authors thank to Mr. Naveen for helping in implementation and simulation of proposed algorithm during experimentation.

\section{REFERENCES}

[1] I.F. Akyildiz, W. Su, Y. Sankarasubramaniam, and E. Cayirci. Wireless sensor networks: a survey. Computer Networks, 38(4):393-422, 2002.

[2] P. Berman, G. Calinescu, C. Shah, and A. Zelikovsky, Power efficient monitoring management in sensor networks, in Proc. of Wireless Communications and Networking Conference, vol. 4. IEEE, March 2004, pp. 2329-2334.

[3] I. Cardei, M. Cardei, (2008) "Energy efficient connected coverage in wireless sensor networks", Int. J. Sen. Netw. Vol. 3, pp 201-210.

[4] M. Cardei, D.-Z. Du, Improving Wireless Sensor Network Lifetime through Power Aware Organization, ACM Wireless Networks, Vol 11, No 3, May 2005.

[5] M. Cardei, M. Thai, Y. Li, and W. Wu, Energy-Efficient Target Coverage in Wireless Sensor Networks, IEEE INFOCOM 2005, Mar. 2005.

[6] M. Cardei, J. Wu, Energy-Efficient Coverage Problems in Wireless Ad Hoc Sensor Networks, accepted to appear 
in Computer Communications, special issue on Sensor Networks.

[7] M. Cheng, L. Ruan, and W. Wu, Achieving Minimum Coverage Breach under Bandwidth Constraints in Wireless Sensor Networks, IEEE INFOCOM 2005, Mar. 2005.

[8] M. Cardei, D. MacCallum, X. Cheng, M. Min, X. Jia, D. Li, D.-Z. Du, (2001) "Wireless sensor networks with energy efficient organization", Journal of Interconnection Networks, vol. 3, pp 213-229.

[9] K. Chakrabarty, S. S. Lyengar, H. Qi, E. Cho, (2002) “ Grid Coverage for Surveillance and Target Location in distributed Sensor Networks", IEEE Transactions on Computers, vol. 51, pp 1448-1453.

[10] J. Carle and D. Simplot, Energy Efficient Area Monitoring by Sensor Networks, IEEE Computer, Vol 37, No 2 (2004) 40-46.

[11] A.J. Goldsmith and S.B. Wicker. Design challenges for energy-constrained ad hoc wireless networks. Wireless Communications, IEEE, 9(4):8\{27, August 2002.

[12] N. Garg, J. K"onemann, (1998) "Faster and simpler algorithms for multicommodity flow and other fractional packing problems". In Proc. 39th Annual Symposium on Foundations of Computer Science (FOCS), pp 300-309.

[13] Liu Hai, P. Wan, C.-W. Yi, Jia Xiaohua, S. Makki, and N. Pissinou. Maximal lifetime scheduling in sensor surveillance networks. In Proc. of INFO-COM 05, volume 4, pages 2482\{2491. IEEE, March 2005.

[14] A. Mainwaring, D. Culler, J. Polastre, R. Szewczyk, and J. Anderson. Wireless sensor networks for habitat monitoring. In Proc. of International Work-shop on Wireless Sensor Networks and Applications, pages $88\{97$. ACM, September 2002.

[15] S. Meguerdichian, F. Koushanfar, M. Potkonjak, and M. Srivastava, Coverage Problems in Wireless Ad-Hoc Sensor Networks, IEEE Infocom 3, pp 1380-1387, 2001.

[16] V. Raghunathan, C. Schurgers, S. Park, and M. B Srivastava, Energy- Aware Wireless Microsensor Networks, IEEE Signal Processing Magazine, 19 (2002), pp 40-50.

[17] S. Slijepcevic, M. Potkonjak, (2001) "Power efficient organization of wireless sensor networks", Proc. of International Conference on Communications (ICC), IEEE, pp 472-476.

[18] D. Tian and N. D. Georganas, A Coverage-Preserving Node Scheduling Scheme for Large Wireless Sensor Networks, Proc. of the 1st ACM Workshop on Wireless Sensor Networks and Applications, 2002.

[19] X. Wang, G. Xing, Y. Zhang, C. Lu, R. Pless, and C. D. Gill, Integrated Coverage and Connectivity Configuration in Wireless Sensor Networks, First ACM Conference on Embedded Networked Sensor Systems, 2003.

[20] J. Wu and S. Yang, Coverage and Connectivity in Sensor Networks with Adjustable Ranges, International Workshop on Mobile and Wireless Networking (MWN), Aug. 2004.

[21] D. Zorbas, D. Glynos, P. Kotzanikolaou, C. Douligeris, (2007) "B $\{\mathrm{GOP}\}$ : An adaptive algorithm for coverage problems in wireless sensor networks", 13th European Wireless Conference, EW 